\section{Gene therapy and the two faces of HIV}

As a human pathogen that threatens public health and economy on a global scale, human immunodeficiency virus (HIV) has been a major target on the international research agenda for therapeutic intervention and eradication. Even in the fledgling field of gene therapy, there are currently a number of clinical protocols in phase $1 / 2$ trials and preclinical studies that target HIV. Lately, however, HIV has emerged as a new player in gene therapy as a gene transfer vector capable of infecting cell types previously thought to be recalcitrant to retroviral vector transduction. As such, it promises to be a valuable new tool for gene therapists. We will review briefly the dual roles of HIV as a target of and tool for gene therapy, and discuss where the two may intersect.

The concept of reconstituting an immune system made up of HIV-resistant cells is theoretically possible by engineering cells to express a foreign gene that interferes with HIV replication. The physical structure of virions and the replication cycle of HIV provide many potential points of attack ${ }^{1,2}$ and it may be safe to say that no stone has been left unturned in this regard. The various strategies have included the use of dominant negative mutants of HIV structural (eg Gag, Env) or essential regulatory (eg Rev) proteins, TAR and rre RNA decoy molecules that act as a 'sink' for the viral regulatory proteins Tat and Rev, intracellular antibodies directed against various viral proteins (eg Env, RT), antisense RNA sequences, and ribozymes designed to cleave at different conserved sites of the HIV genome. In addition, ribozymes directed against cellular proteins important to the HIV replication cycle have also been shown to inhibit HIV infection. Currently, one of the best examples of a suitable cellular target is the chemokine receptor CCR5, which is used as a co-receptor during HIV entry into cells. People completely lacking a functional CCR5 (homozygous $\Delta 32$ deletion) are not only healthy, but also relatively resistant to HIV infection, while those with a heterozygous deletion generally display a slower course of progression to AIDS after infection with HIV. Thus, it is possible that down-regulation of CCR5 expression by ribozyme gene transfer could result in a clinical benefit.

There are clinical protocols that have been completed or are on-going to determine the safety and feasibility of transferring various antiviral genes (transdominant Rev, hairpin ribozyme, hammerhead ribozyme, antisense RNA) into either mature $\mathrm{T}$ cells or hematopoietic precursor $\left(\mathrm{CD} 4^{+}\right)$cells followed by reinfusion of autologous transduced cells into patients. The antiviral genes were either in the form of DNA or expressed in murine retrovi- ral vectors. The results so far show that, in general, such procedures are safe and that $\mathrm{T}$ cells harboring the antiviral gene(s) survived preferentially over those containing a control vector, presumably due to resistance to cytopathic HIV infection. However, major limitations must be overcome before turning these gene transfer studies into real therapies: the efficiency of gene transfer, the number of transduced cells infused relative to total untransduced cells, and persistent expression of the transgene. Furthermore, protocols that aim at $\mathrm{T}$ cell transduction need to address the issues of cellular turnover and the existence of other target cells for HIV, such as macrophages and dendritic cells. With $\mathrm{CD}^{+} 4^{+}$cell transduction, engraftment and persistence of the transduced cells has been suboptimal, and there is yet no evidence that the true 'stem' cells have been successfully transduced.

Some of the above limitations stem from the lack of optimal gene transfer vectors. Murine retroviral vectors are widely used because of their ability to integrate stably into target cells and low immunogenicity. However, they can only transduce actively dividing cells, and are therefore unable to access cells like terminally differentiated macrophages or hematopoietic stem cells, which are not actively dividing most of the time. Here is where HIV enters again, but cast in a very different role. ${ }^{3}$ The realization that HIV can efficiently infect nondividing or terminally differentiated cells opened up the possibility that HIV vectors might do likewise and therefore be useful in gene transfer studies.

Initially, vector systems derived from HIV-1 were developed to facilitate the study of viral replication and pathogenesis. Development of such vector systems was problematic, due to the presence of viral accessory genes and the difficulty in establishing stable packaging cell lines, possibly because of potential toxicity of certain viral proteins. Eventually, however, reliable vector systems using transient transfection of vector and packaging plasmids were developed. The use of a heterologous virus envelope (eg VSV-G), rather than the native HIV envelope glycoprotein, to pseudotype vector particles also greatly expanded the range of cell types that the vectors can transduce. Pseudotyped HIV-1 vectors have been shown to transduce efficiently growth arrested cells, macrophages, dendritic cells, neuronal cells, and unstimulated $\mathrm{CD} 4^{+}$cells in vitro, ${ }^{4}$ and the brain, liver and muscle in vivo. ${ }^{5}$ In parallel, efforts were made to increase the safety of the vectors, starting with removal of the viral accessory genes.

Although the ability of HIV to infect nondividing cells has been variably attributed to the nuclear localization signal (nls) in the matrix (MA) protein, Vpr, or integrase 
(IN), the mechanism of infection of nondividing cells has yet to be fully elucidated. Recently, it was found that the central polypurine tract (cPPT) present in HIV pol increases the efficiency of nuclear translocation of HIV vectors. ${ }^{6}$ In any event, HIV packaging systems lacking all accessory genes still yield vector virions capable of infecting nondividing cells. This has simplified vector production and decreased the chances of replication-competent retrovirus generation during vector production. Third or fourth generations of 'new and improved' vectors designed to minimize virus recombination, eg substitution of CTE for rre elements, deletion of U3 sequences (SIN vectors), and further separation of the packaging genes on different plasmids, are being developed. Another option to increase the safety (or at least the perception of safety) of lentiviral vectors is to employ lentiviruses other than HIV-1, eg HIV-2, feline immunodeficieny virus (FIV), and equine infectious anemia virus (EIAV). ${ }^{3}$ These viruses have less or no pathogenicity in humans, and seroconversion to the non-primate lentiviral proteins will carry fewer stigmas than seroconversion to the human pathogens. Furthermore, HIV-1 vectors may be incompatible with the expressed therapeutic genes (eg those that target HIV-1), and in such cases use of these other lentiviral vectors may be more appropriate.

Although the lentiviral vectors are expected to become valuable workhorses of gene therapy some day, one must still overcome the psychological barrier of administering a live HIV-1 vector (however attenuated) into noninfected patients. Therefore, one would imagine that the first test of principles for the use of lentiviral vectors in clinical gene therapy would be in HIV-infected populations. In these patients, the remote risk of replicationcompetent retrovirus regeneration would be a moot issue. It is difficult to conceive of more deleterious recombinants between vector and helper virus than the HIV quasi-species already existing in the patient. Furthermore, the possibility of vector mobilization (dissemination of the therapeutic vector to additional HIV target cells) might be viewed as an asset rather than a risk. However, as mentioned above, the use of HIV-1 vectors to deliver anti-HIV-1 genes may be self-defeating, and here an HIV-2 vector might be preferable. Finally, HIV-1-based vectors may be viewed as an extreme form of attenuated virus that can yield one round of infection, persistent expression of select viral antigens, and no productive virus. As such, they may be designed to be immunizing vectors that can target antigen-presenting cells. HIV-1 vectors expressing Gag-Pol antigens have been shown to transduce efficiently dendritic cells, which in turn can immunize $\mathrm{T}$ cells to exhibit virus-specific cytotoxicity in vitro. ${ }^{7}$ It is conceivable that lentiviral vectors can be used to deliver other antigens (eg tumor antigens) for use in gene transfer approaches to treatment of diseases other than HIV infection.

Thus, HIV sits in the unusual position of being a major human pathogen, a potential target for gene transfer therapy, and a potential tool for use in gene transfer applications. One might argue that gene therapy is not practical for HIV disease, especially in view of the many treatment options now possible, because the cost alone of gene transfer and stem cell transplantation would be prohibitive. However, current treatments for HIV infection have their drawbacks (eg cost, availability, patient noncompliance, side-effects, virus resistance), and we anticipate that gene therapy would be more accessible and affordable to the general public as the technology is improved. The recent report demonstrating apparent successful gene transfer for treatment of severe combined immunodeficiency-X1 $\quad\left(\right.$ SCID-X1) ${ }^{8}$ illustrates how incremental improvements in gene therapy strategies, both at the basic science and clinical level, can be combined to overcome successfully what at times appear to be insurmountable obstacles. Development of lentiviral vectors should represent another step forward in the long path of gene therapy technology development.

F Wong-Staal ${ }^{1}$ and GL Buchschacher $\mathrm{Jr}^{2}$ ${ }^{1}$ Departments of Medicine and Biology and ${ }^{2}$ Division of Hematology/Oncology, Department of Medicine University of California, San Diego La Jolla, California 92093-0665

USA

\section{References}

1 Buchschacher GL Jr. Molecular targets of gene transfer therapy for HIV infection. JAMA 1993; 269: 2880-2886.

2 Poeschla EM, Wong-Staal F. Gene therapy and HIV disease. In: Volberding P, Jacobson MA (eds). AIDS Clinical Review. Marcel Dekker: New York, 1995, pp 3-45.

3 Buchschacher GL Jr, Wong-Staal F. Development of lentiviral vectors for gene therapy for human diseases. Blood 2000; 95: 2499-2504

4 Miyoshi $\mathrm{H}$ et al. Transduction of human CD34+ cells that mediate long-term engraftment of NOD/SCID mice by HIV vectors. Science 1999; 283: 682-686.

5 Naldini L et al. In vivo gene delivery and stable transduction of nondividing cells by a lentiviral vector. Science 1996; 272: 263267.

6 Zennou $\mathrm{V}$ et al. HIV-1 genome nuclear import is mediated by a central DNA flap. Cell 2000; 101: 173-185.

7 Gruber A et al. Dendritic cells transduced by multiply deleted HIV-1 vectors exhibit normal phenotypes and functions and elicit an HIV-specific CTL response in vitro. Blood (in press).

8 Cavassana-Calvo $M$ et al. Gene therapy of human severe combined immunodeficiency (SCID)-X1 disease. Science 2000; 288: 669-672. 